\section{METAL MAGNETISM}

\section{Magnetism and Metallurgy}

Edited by Ami E. Berkowitz and Eckart Kneller. Vol. 1. Pp. xiii $+512+39$. 175s. Vol. 2. Pp. xiv +513-838 +32. 159s. (Academic Press: New York and London, January 1970.)

THEse two volumes contain articles on topics selected from the magnetic properties of metals and provide a stimulating and up to date review of the field. The individual articles are written by authors who are experts in their chosen subject matter and are written with considerable care and authority. The level of writing is such that a third year undergraduate in the United Kingdom will be able to find a great deal of interest in the books, while a first year postgraduate student will find that these articles provide a most valuable general introduction to his research if this is in the field of metal magnetism. The industrial scientist will also find in the different articles not only reviews which may be of a direct relevance to his work in progress, but also attractive and readable surveys which will serve well as an introduction to, or a revision of, material with which he would like to become familiar.

The topics covered in the volumes are wide ranging. The introductory section "Principles of Magnetism" begins with an article at undergraduate level on basic magnetic theory and is followed by a somewhat more advanced treatment of energy relations, with a brief mention of domain structure and magnetization processes. The third article in this section gives an elementary account of magnetic resonance phenomena. The second section reviews "Experimental Methods". This section contains two extremely useful articles covering magnetization, anisotropy, magneto-optical, and hysteresis effects. The third and largest section occupies the latter part of volume one and the whole of volume two. Thus, whereas the first two sections each consist of about 100 pages of text, this section involves about 600 pages. The title of this third section is the "Relation between Magnetic and Structural Properties". It contains twelve articles on topics such as "Magnetic Moments and Transition Temperatures" by E. Vogt, "Constitution of Multiphase Alloys" by A. E. Berkowitz, "Fine Particle Theory" by E. Kneller, "Alnico Permanent Magnet Alloys" by K. J. de Vos, "Effects of Atomic Order-Disorder on Magnetic Properties" by J. S. Kouvel, "Textured Magnetic Materials" by C. D. Graham, jun., and "Interpretation of Magnetic Resonance Measurements in Metals" by D. S. Rodbell. For the most part these are review articles at postgraduate level.

The books are well printed and the individual articles contain clear diagrams and useful lists of references. In summary, these two volumes can be recommended to any group interested generally in the field covering the properties of magnetic metals.

D. M. S. BAGguleY

\section{EPR AND IONS}

Electron Paramagnetic Resonance of Transition lons Bv A. Abragam and B. Bleaney. (International Series of Monographs on Physies.) Pp. xiv +911. (Clarendon Press: Oxford; Oxford University Press: London, April 1970.) 260s.

ALTHOUGH a considerable volume of literature exists on electron paramagnetic resonance of transition metal ions, there has up to now been no standard text which deals comprehensively with both the theoretical and experimental aspects of the subject. The book is thus very welcome.

The first of the three sections of the book gives a preliminary survey of the field of electron paramagnetic resonance (EPR) with the aim of introducing a beginner to the many concepts which form the basis of the subject.
The second and largest section gives a general survey covering the resonance phenomenon, the interpretation of spectra using spin Hamiltonians, electron-nuclear double resonance and the effects of spin-spin and spin-phonon interactions. Four very valuable chapters in this section are devoted to a systematic study of the properties of the 4f, 5f and 3d ions in weak and intermediate strength ligand fields of predominantly octahedral symmetry and of all d-group ions in strong octahedral fields. Brief mention is also made of tetrahedral and cubal fields for d-group ions. The final section of the book is devoted to a theoretical survey of the fundamentals of transition metal ions in crystals relevant to resonance spectroscopy. The chapters on group theory outline the basic concepts and discuss in some detail the properties of the rotation and cubic groups and they are followed by a study of time reversal and Kramers degeneracy. These basic ideas are used in detailed studies of crystal field theory, hyperfine interactions, the formation of covalent bands and the Jahn-Teller effect. Finally, extensive tables in the appendix contain a great deal of valuable information.

One outstanding feature of the book is that it will undoubtedly be useful for the beginner, the experimentalist working in EPR, and also the theoretician. Much of the theoretical section is also applicable to a wider field such as optical and infrared spectroscopy of ions, acoustic paramagnetic resonance and phonon spectroscopy. Because, however, the book is divided into three parts, it is inevitable that the development of the subject is not always logical and it is sometimes difficult to know where to find certain topics. Referencing to original work is not as comprehensive as usual in a book of this nature but this is probably balanced by the inclusion of much unpublished material.

The production of the book is very good, although back referencing would have been greatly assisted by printing the section numbers at the top of each double page. I feel sure that the book will prove a most valuable source of information for everybody in the field. It is a great pity that its use would seem to be limited by its price.

\section{A. Bates}

\section{MICROSCOPICAL TECHNIQUE}

\section{Field Ion Microscopy}

Principles and Applications. By Erwin W. Müller and Tien Tzou Tsong. Pp. ix +314. (Elsevier: New York, London and Amsterdam, 1969.) $200 s$.

THIs is the first book to appear in which an attempt is made to expound both the principles and applications of field-ion microscopy $a b$ initio. The emphasis is, however, on the theoretical and experimental basis of the technique; less than a quarter of the book is devoted to applications. After a short historical introduction there are involved chapters, at an advanced level, which describe the present incomplete understanding of the field ionization and field evaporation processes. The highlights of the book are the chapters on design of equipment and ingenious experimental techniques, many of which were developed in the author's laboratory. Consequently there is a wealth of experimental detail much of which would otherwise have remained unremarked and unpublished.

The rather limited amount of space devoted to applications and methods of image interpretation has resulted in a sparse coverage of these topics. It might have been desirable in this part to include at least more references, especially concerning subjects such as the image contrast from alloys where controversy still exists.

There is a useful index and the book is profusely illustrated, but the small size of some of the figures detracts from their clarity.

The book is generally accurate, although the initiate will be amused to learn how to make specimens of titanium 